\title{
Direct mapping of polarization fields from STEM images: A Deep Learning based exploration of ferroelectrics
}

Ayana Ghosh ${ }^{1,2}$, Christopher Nelson ${ }^{1}$, Mark Oxley ${ }^{1}$, Xiaohang Zhang ${ }^{3}$, Maxim Ziatdinov ${ }^{1,2}$, Ichiro Takeuchi $^{3}$ and Sergei Kalinin ${ }^{1}$

${ }^{1}$ Center for Nanophase Materials Sciences, Oak Ridge National Laboratory, Oak Ridge, Tennessee, United States, ${ }^{2}$ Computational Sciences and Engineering Division, Oak Ridge National Laboratory, Oak Ridge, Tennessee, United States, ${ }^{3}$ University of Maryland, Maryland, United States,

Scanning transmission electron microscopy (STEM) has emerged as a powerful tool over the last decade for probing the atomic structures of complex materials with picometer precision. This has unlocked the gateway towards exploring functional properties such as ferroelectricity, ferroelasticity, and chemical phenomena on the atomic scale. The well-established approach ${ }^{1-8}$ to quantitively analyze such images involve finding atom positions or atomic columns corresponding to A-site and B-site cations and account for atomic displacements to retrieve information about order parameter fields such as polarization. Polarization maps retrieved utilizing the traditional approach for a $[100]_{\text {psuedocubic }} \mathrm{BiFeO}_{3}(\mathrm{BFO})$ film on $\mathrm{SrTiO}_{3}$ (STO) substrate is shown in Figure 1.

However, this process of parameterization of all atoms and consequently mapping of polarization fields is highly labor intensive. The ad-hoc Gaussian fitting to position the atomic column center and mis-tilts in measurements also lead to systematic errors in the polarization values.Hence, an alternate approach becomes crucial to not only bypass these shortcomings but also facilitate rapid transformation of STEM images for paving the pathway to develop physics-informed analyses and automated experiments.

In this work, we have proposed an alternate approach of utilizing deep convolution neural networks (DCNNs) for direct polarization mapping from STEM data. The network is trained on the labeled part of the image (i.e., for human labelling), and the trained network is subsequently applied to other images. This framework shows how to extract polarization maps without atom-finding and also compare the results with that performed using the traditional approach. All the networks are constructed using subimages (parts of original images cropped for a specific window size) generated using atom finding and non-atom finding methods. These are the local features for the DCNNs and the corresponding polarization values become the target set. We have streamlined the workflow with a number of STEM images with various Samarium (Sm) dopant concentrations in BFO, such that the explored feature space encompasses both ferroelectric and weakly-ferroelectric systems.

Figure 2 (I) shows the key steps outlining the DCNN analysis and (II - III) are the representative subimages utilized to build the models. The ground truth along with predictions using non-centered subimages and errors of polarization maps for $0 \%$ Sm-doped rhombohedral BFO, $7 \%$ and $10 \%$ Sm-doped monoclinic BFO are shown in Figure 2 (a-i), respectively. Further analysis investigating noise-window size sensitivity cross training on one composition of ferroelectric system and applying to another, feature maps carrying information about encoded features and layer by layer visualizations have also been integrated in the study. In addition, to gain insight into the possible origins of the observed behaviors, including asymmetric polarization distributions and cross-training, we have simulated ADF-STEM images for several tilt values off from the zone axis. These simulations quantify the A-site versus B-site cation shifts in the ferroelectric systems which would result in an apparent change of polarization. 
In summary, we have developed a framework for the analysis of atomically resolved STEM image data of ferroelectric materials to extract local polarization based on sub-image analysis. We demonstrate that the application of DCNN-based regression on sub-images centered on a given sub-lattice yields values similar to direct column position analysis. Hence, this study reveals the tremendous potential of the DCNN for the analysis of high-resolution STEM imaging and spectral data and highlights the associated limitations.

\section{Acknowledgments:}

This effort (STEM) is based upon work supported by the U.S. Department of Energy (DOE), Office of Science, Basic Energy Sciences (BES), Materials Sciences and Engineering Division (S.V.K., C.T.N.) and was performed and partially supported (M.Z.) at Oak Ridge National Laboratory's Center for Nanophase Materials Sciences (CNMS), a U.S. DOE, Office of Science User Facility.This effort (ML) is based upon work supported by the U.S. DOE, Office of Science, Office of Basic Energy Sciences Data, Artificial Intelligence and Machine Learning at DOE Scientific User Facilities (A.G.). The work at the University of Maryland was supported in part by the National Institute of Standards and Technology Cooperative Agreement 70NANB17H301 and the Center for Spintronic Materials in Advanced Information Technologies (SMART) one of the centers in nCORE, a Semiconductor Research Corporation (SRC) program sponsored by NSF and NIST.

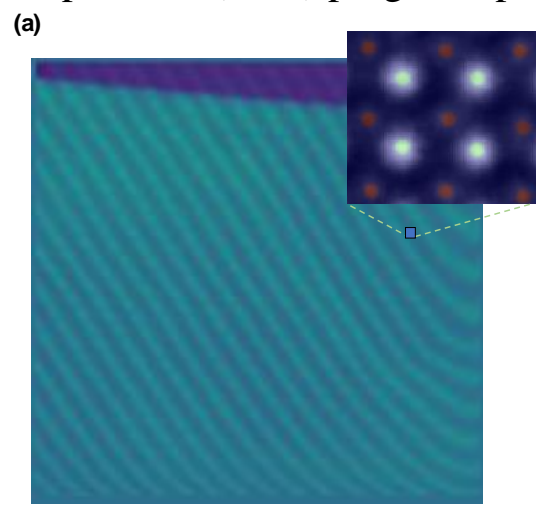

(b)

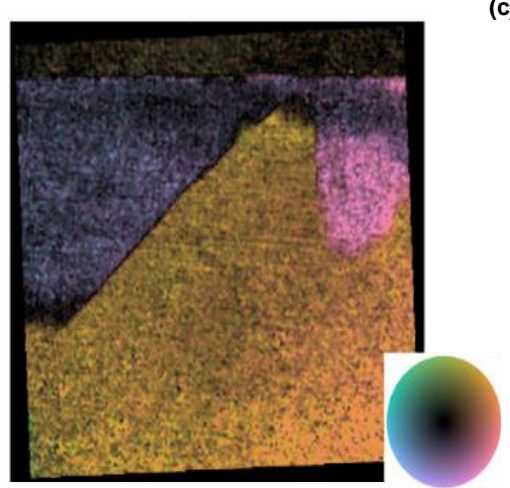

(c)

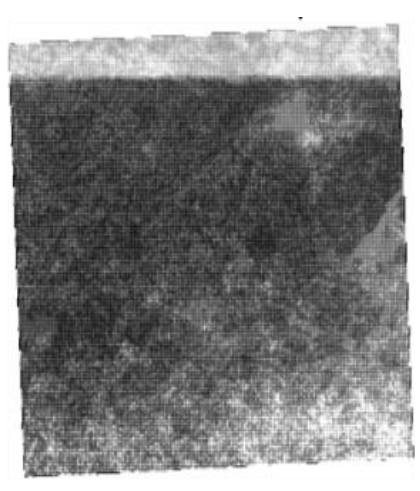

Figure 1. Polarization mapping via atomic position parametrization. a) HAADF-STEM image of a $[100]_{\text {psuedocubic }}$ BFO film on STO substrate with gaussian fits corresponding to inset region. Polar displacement, $\mathrm{P}$, defined as the vector between central Fe position and average Bi position (blue cross). $\mathrm{P}$ distribution map (b) corresponding to HAADF-STEM image in (a) with highlighted principal features and domain walls. Color legend maximum radius corresponds to 50pm. Distribution of $\mathrm{P}$ uncertainty is displayed in (c). Scalebar is $5 \mathrm{~nm}$. 


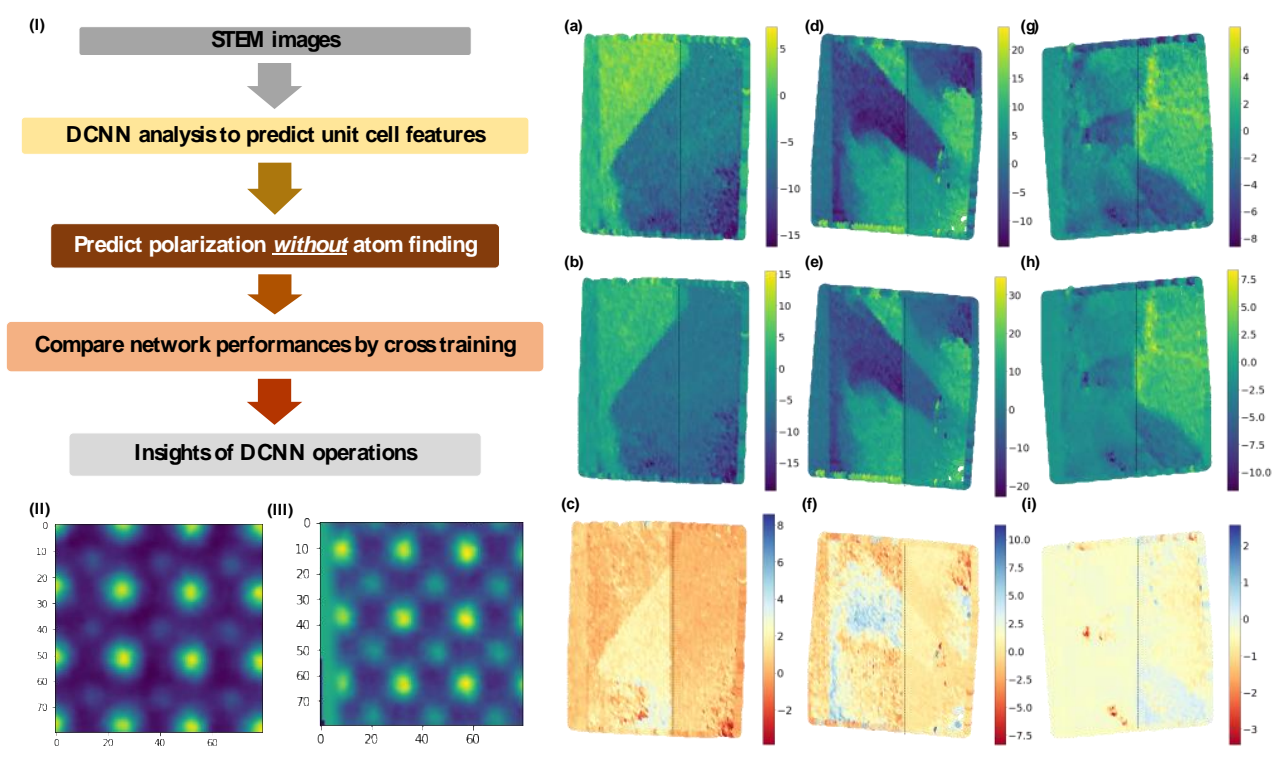

Figure 2. (I) High-level schematic showing the DCNN-STEM framework. Representative subimages generated with (II) and without (III) atom finding approaches are shown for comparison. Polarization maps (using without atom finding method) representing the ground truth (top row), prediction (middle row), and differences between them (bottom row) are shown for $0 \%$ (a-c), $7 \%$ (d-f) and 10\% (g-i) Smdoped BFO, respectively. Predictions obtained using three different networks as trained on $2 / 3$ of the full stack of sub-images (for every concentration) and tested on the rest. Vertical line in plots refer to the traintest splits.

\section{References}

1. Zubko, P. et al., In Annual Review of Condensed Matter Physics, Vol 2, Langer, J. S., Ed. Annual Reviews: Palo Alto, 2011; Vol. 2, pp 141-165.

2. Chisholm, M. F. et al., Atomic-Scale Compensation Phenomena at Polar Interfaces. Phys. Rev. Lett. 2010, 105 (19).

3. Chang, H. J. et al., Atomically Resolved Mapping of Polarization and Electric Fields Across Ferroelectric/Oxide Interfaces by Z-contrast Imaging. Adv. Mater. 2011, 23 (21), 2474-+.

4. Borisevich, A. Y. et al., Interface dipole between two metallic oxides caused by localized oxygen vacancies. Phys. Rev. B 2012, 86 (14).

5. Borisevich, A. Y. et al., Exploring Mesoscopic Physics of Vacancy-Ordered Systems through Atomic Scale Observations of Topological Defects. Phys. Rev. Lett. 2012, 109 (6).

6. Li, Q. at al., Quantification of flexoelectricity in PbTiO3/SrTiO3 superlattice polar vortices using machine learning and phase-field modeling. Nat. Commun. 2017, 8.

7. Ziatdinov, M. et al., Causal analysis of competing atomistic mechanisms in ferroelectric materials from high-resolution scanning transmission electron microscopy data. npjComput. Mater. 2020, 6 (1), 9.

8. Ziatdinov, M. et al., Deep Learning of Atomically Resolved Scanning Transmission Electron Microscopy Images: Chemical Identification and Tracking Local Transformations. ACS Nano 2017, 11 (12), 12742-12752. 